\title{
Estado de salud mental de personas víctimas del conflicto armado. Caso Chengue, Sucre-Colombia
}

\author{
Andrés Fernando Ramírez Giraldo1, Olga Leonor Hernández Bustamante ${ }^{2}$ \\ Kelly Romero Acosta ${ }^{3}$, Eduardo Porras Mendoza ${ }^{4}$
}

\section{Resumen}

Para esta investigación se trabajó con víctimas del conflicto armado del Corregimiento de Chengue, en el Municipio de Ovejas. Estas personas fueron víctimas del conflicto armado. Los habitantes de la población de Chengue se desplazaron lejos de sus hogares debido a una masacre. El objetivo de este estudio es describir el estado de salud mental en personas oriundas del corregimiento de Chengue. Esta investigación se hizo desde un paradigma cuantitativo de nivel descriptivo y de corte transversal; se aplicó una entrevista diagnóstica estructurada de breve duración, que explora los principales trastornos psiquiátricos del Eje I del DSM-IV. En total participaron 61 personas adultas del corregimiento de Chengue. Entre los resultados se encontró que los trastornos presentes más altos en la población de Chengue son la depresión mayor actual (39,3\%), Trastorno de estrés postraumático $(26,2 \%)$ y trastorno de ansiedad generalizada (24,6\%); esto muestra a una población preocupada tanto por el pasado como por su futuro.

Palabras clave: Estado de salud mental, desplazamiento armado, masacre, Chengue.

\footnotetext{
1 Doctor en Psicología con orientación en neurociencias cognitivas Universidad Maimónides. Magíster en neuropsicología. Psicólogo. Director del grupo Dimensiones Humanas. Docente investigador CECAR.

2 Magíster en psicología. Especialista en derechos humanos. Especialista en psicología clínica. Especialista en docencia. Docente CECAR.

3 Doctora en Psicología de niños, adolescentes y adultos. Magíster de psicopatología infanto-juvenil de la Universidad Autónoma de Barcelona.

4 Abogado Universidad Libre (Barranquilla). Magíster en Historia Universidad Pedagógica y Tecnológica de Colombia-UPTC (Tunja). Trabajo de grado: Memoria Histórica de Chengue, 1990-2012. Sociedad, Cultura y Violencias en los Montes de María (Nota: Laureado).
} 


\section{INTRODUCCIÓN}

Para llevar a cabo esta investigación se trabajó específicamente con miembros del corregimiento de Chengue en el Municipio de Ovejas. El caso Chengue es la vivencia de un hecho violento que abruptamente finalizó en un desplazamiento forzado.

Según el informe $n^{\circ}$ 45/07 de la Comisión Interamericana de Derechos Humanos (2007), el día 17 de enero del 2001, en la madrugada, un grupo de autodefensas (AUC) ingresó a Chengue y durante dos horas perpetraron una serie de actos de tortura, homicidio y desaparición.

Los habitantes de la población relataron a los investigadores que una madrugada, un grupo de hombres armados, sacaron a varios vecinos de Chengue de sus casas pues supuestamente se necesitaba verificar su nombre en un computador. De estos hombres, 27 fueron asesinados por un golpe de martillo en sus cabezas. Los sobrevivientes (hombres, mujeres, niños y niñas) escaparon hacia Montes de María, hasta llegar al municipio de Ovejas. Mientas ellos escapaban, los hombres armados prendieron fuego a las casas y huyeron. Ese mismo día los pocos hombres que volvieron al pueblo encontraron 27 hombres muertos; debido a este hecho, la mayoría de habitantes de Chengue se desplazó forzosamente a pueblos vecinos.

El desplazamiento forzado se caracteriza por ser un fenómeno socio-político que produce un deterioro en la calidad de vida de las personas. Según Deng (1988), esta situación puede provocar, entre otras cosas, pérdida de oportunidades educativas, separación de los miembros de la familia, destrucción de empleos y problemas para acceder a necesidades básicas, tales como los servicios de salud (Deng, 1998). Y es que los desplazados, cómo en el caso Chengue, no sólo sufren por el desplazamiento del lugar de origen a cualquier otra parte, sino que, además, sufren por situaciones dolorosas que podrían implicar amenazas, muerte, reclutamiento de familiares, entre otros (Juárez y Guerra, 2011).

Cabe resaltar que, en muchos casos, la situación que se tenía antes del acto violento que provocó el desplazamiento, podía ser ya precaria. Esto provocaría en estos casos, un proceso de desarraigo que implicaría más pérdidas (Alejo, 2005). Estas pérdidas vienen acompañadas por un conjunto de acontecimientos vítales estresantes que pueden desencadenar la aparición de trastornos mentales (Buendía \& Mira, 1993; Falla, Chávez \& Molano, 2005; Martín \& Ochotorena, 2004; Médicos sin fronteras, 2006; Palacios \& Heinze, 2002; Santacruz \& Ardila, 2002). En efecto, estudios anteriores indican que la desaparición de conocidos produce un nivel de 
sufrimiento tal, que se puede manifestar en somatizaciones y trastornos alimenticios (Sacipa, 2003).

Botelho y Conde (2011) aseguran que el impacto emocional que tienen los desplazados puede constituirse en un factor de riesgo para el desarrollo de trastorno de estrés post-traumático (TEPT). En esta línea, Echenique, et al. (2008) hallaron que $5.5 \%$ de 142 sujetos desplazados se vieron afectados por TEPT en el momento de la aplicación del cuestionario. Todos los sucesos violentos que vivieron por motivo del desplazamiento, pudieron haber influido en la aparición del trastorno, sin ninguna distinción de características socio-demográficas.

¿Puede ser esta la razón por la cual los colombianos presentan una alta prevalencia de trastornos mentales? Estudios anteriores han señalado que en Colombia existe una alta prevalencia de trastornos mentales. En el año 2003 se realizó un estudio de salud mental a nivel nacional y se halló que el $40.1 \%$ de los participantes presentaron al menos un trastorno mental durante su vida (Ministerio de Protección Social, 2005). Los trastornos del estado de ánimo y los trastornos de ansiedad fueron los más prevalentes en la muestra estudiada.

Posteriormente, en el año 2012 se publicaron los resultados del primer estudio poblacional de salud mental de la ciudad de Medellín (Torres, et al., 2012). Los resultados indicaron que de ,81\% a 10,7\% de los encuestados presentaron algún trastorno de ansiedad; asimismo, de ,55\% a 5,14\% presentaron algún trastorno del estado de ánimo; y que de ,60\% a 4,89\% presentaron algún trastorno por uso y abuso de sustancias.

El estudio más reciente que se ha publicado sobre la prevalencia de trastornos mentales en la población colombiana se publicó en el 2015. Entre los resultados se halló una prevalencia de trastornos del estado de ánimo de entre $0,3 \%$ y $1,9 \%$. Los trastornos afectivos (trastorno bipolar I y II, cualquier otro trastorno afectivo) se encontraron entre 0.1 y $2.4 \%$ de la muestra estudiada, y los de ansiedad de 0,1\% a 9,5\%.

Por su parte, Gómez (2001), señala que en Colombia alrededor del $20 \%$ de la población colombiana padece angustia elevada relacionada con condiciones económicas, sociales, culturales y políticas a las que se les tiene que agregar el conflicto armado. Cinco años después un informe de Médicos sin Fronteras (2006) señala que existe una prevalencia de $27,6 \%$ de trastornos mentales en ciertas poblaciones desplazadas.

En efecto, el conflicto armado y el desplazamiento forzado pueden haber dejado secuelas emocionales en sus miles de afectados. Por esta razón, es importante que se realicen estudios que identifiquen el estado de salud mental de los afectados del conflicto colombiano. Siendo así, el ob- 
jetivo de este estudio es identificar el estado de salud mental en personas víctimas del conflicto armado en Chengue.

\section{MÉTODO}

Esta investigación estuvo enmarcada en un paradigma cuantitativo de tipo observacional, de un nivel descriptivo y de un corte trasversal, en una población de víctimas del conflicto armado colombiano de la región Caribe. El muestreo es intencional, ya que son personas reconocidas como víctimas y que vivieron la situación directamente de desplazamiento.

\section{Participantes y procedimiento}

El proceso investigativo se llevó a cabo, entre el mes de junio y julio de 2008. Se entrevistó a 61 personas, 23 (37.7\%) mujeres y 38 (62.3\%) hombres, de la comunidad de Chengue residentes en Ovejas, que se habían traslado a esta localidad luego de la masacre de 2001. Esta investigación tiene el apoyo del Comité Científico de la Corporación Universitaria del Caribe, CECAR. A todos los participantes se les pidió firmar el consentimiento informado y todos aceptaron participar.

\section{Instrumentos}

Mini Internacional Neuropsiquiátrica Evaluación (Sheehan, et al., 1997; Sheehan, et al., 1998). Es una entrevista diagnóstica estructurada de breve duración que explora los principales trastornos psiquiátricos del Eje I del DSM-IV y de la CIE-10. Esta prueba está dividida en 16 módulos, los cuales están referenciados por una letra correspondiente a una categoría diagnóstica. La respuesta a los ítems es dicotómica es decir, Sí o NO y la marcación de las respuestas por el evaluador, depende de un juicio clínico. Su administración dura alrededor de 15 minutos.

\section{Análisis Estadístico}

Para el análisis estadístico se utilizó el Statistical Package for Social Science (SPSS v. 20), con el fin de calcular frecuencias y porcentajes de los casos estudiados.

\section{Resultados}

\section{Trastornos del estado de ánimo}

Se halló entre 1,6\% y 39,3\% de trastornos del estado de ánimo en la muestra, mostrando el episodio mayor con casi $40 \%$ de la población afectada (ver tabla 1). 
Tabla 1. Prevalencia de trastornos del estado de ánimo en la muestra

\begin{tabular}{|c|c|c|}
\hline Lista de trastornos & Frecuencia & Porcentaje \\
\hline Episodio depresivo Mayor Actual & 24 & 39,3 \\
\hline Episodio depresivo Mayor Pasado & 9 & 14,8 \\
\hline Distimia & 5 & 8.3 \\
\hline Episodio Hipomaniaco Actual & 1 & 1,6 \\
\hline Episodio Hipomaniaco Pasado & 1 & 1,6 \\
\hline Trastorno del estado de animo & 1 & 1,6 \\
\hline
\end{tabular}

\section{Trastornos de ansiedad}

Se halló entre 1.6 y 26,2\% de trastornos de ansiedad, siendo el más prevalente el trastorno de estrés postraumático. La tabla 2 muestra esta información más ampliamente.

Tabla 2. Prevalencia de trastornos de ansiedad

\begin{tabular}{|c|c|c|}
\hline Lista de trastornos & Frecuencia & Porcentaje \\
\hline Trastorno de Angustia Alguna vez & 11 & 18,0 \\
\hline $\begin{array}{c}\text { Trastorno de Angustia con crisis limitadas } \\
\text { actual }\end{array}$ & 4 & 6,6 \\
\hline Trastorno de Angustia Actual & 7 & 11,5 \\
\hline Agorafobia & 1 & 1,6 \\
\hline Episodio de angustia sin agorafobia actual & 3 & 4,9 \\
\hline Episodio de angustia con agorafobia actual & 3 & 4,9 \\
\hline Episodio de angustia sin historial de \\
agorafobia & 3 & 4,9 \\
\hline Fobia Social & 1 & 1,6 \\
\hline Trastorno Obsesivo- Compulsivo & 3 & 4,9 \\
\hline Trastorno de estrés-postraumatico & 16 & 26,2 \\
\hline Trastorno de ansiedad generalizada & 15 & 24,6 \\
\hline
\end{tabular}

\section{Trastornos por consumo de alcohol}

En la muestra se halló que una sola persona presenta trastorno por consumo de alcohol con dependencia y abuso. Para más información ver la tabla 3. 
Tabla 3. Trastornos por consumo de alcohol

\begin{tabular}{|c|c|c|}
\hline Lista de trastornos & Frecuencia & Porcentaje \\
\hline $\begin{array}{c}\text { Trastorno por consumo de alcohol con } \\
\text { dependiencia }\end{array}$ & 1 & 1,6 \\
\hline Trastorno por consumo de alcohol con abuso & 1 & 1,6 \\
\hline
\end{tabular}

Trastornos psicóticos

Se encontró una prevalencia de 3,3\% $(n=2)$ de trastorno psicótico actual y $1,6(n=1)$ de trastorno psicótico de por vida

\section{DISCUSIÓN}

La unidad para las víctimas en Colombia regraba para el año 2015 7.438.023 personas en el Registro Único de Víctimas, (RUV, 2017) registra 8.048.252 víctimas del conflicto armado; esto confirma lo planteado por Amnistía Internacional (2004), cuando dice que Colombia presenta una de las tasas de desplazamiento más altas del mundo mencionan que en un lapso de 20 años (1985-2005) se desplazaron más de tres millones de personas. El tener que desplazarse puede generar altos niveles de estrés en los diferentes ciudadanos (Martín \& Ochotorena, 2004; Buendía \& Mira, 1993) y esto llevar a presentar diferentes trastornos psicológicos. El objetivo de este estudio fue identificar los diferentes trastornos psicológicos presentes en individuos que vivieron un hecho violento causado por un grupo armado ilegal que terminó en desplazamiento.

Es importante destacar en los resultados encontrados en esta investigación que los sujetos víctimas de desplazamiento, además de haberles tocado vivenciar una experiencia de matanza de algunos amigos y familiares, presentan una alta prevalencia de episodio depresivo mayor en la actualidad y alta prevalencia de TEPT y ansiedad generalizada, coherente con lo hallado en otras investigaciones de población con características similares (Martín \& Ochotorena, 2004; Palacio, Mandariaga \& Sabatier, 2001; Silove, Steel, McGorry \& Mohan, 1998). Por lo tanto, podríamos inferir que las comunidades que fueron víctimas del conflicto y haber vivenciado experiencias significativas en su victimización (como masacre, desplazamiento, constreñimiento, extorsiones, amenazas y violencia sexual) puede explicar las características presentes de depresión y estrés postrauma presentes.

La característica esencial del trastorno por estrés postraumático es la aparición de síntomas característicos que sigue a la exposición a un acontecimiento estresante y extremadamente traumático, donde el individuo se ve envuelto en hechos que representan un peligro real para su vida o cual- 
quier otra amenaza para su integridad física (DSM 5, 2013); La respuesta del sujeto a este acontecimiento debe incluir temor, desesperanza y horrores intensos; el cuadro sintomático característico secundario a la exposición al intenso trauma debe incluir la presencia de reexperimentación persistente del acontecimiento traumático, de evitación persistente de los estímulos asociados a él y el embotamiento de la capacidad de respuesta del individuo, y de síntomas persistentes de activación (DSM IV -TR, 2000). El trastorno puede llegar a ser especialmente grave o duradero cuando el agente estresante es obra de otros seres humanos (p. ej., torturas, violaciones). La probabilidad de presentar este trastorno puede verse aumentada cuanto más intenso o más cerca físicamente se encuentre el agente estresante.

Los resultados de la entrevista muestran que en este trastorno se necesita urgente atención, ya que de la población total evaluada presenta riesgo de padecer TEPT el $26,2 \%$, en contraste con la media en Colombia donde se estima que su presencia es del 1,8\% (Posada, et al., 2004) ० 3,3\% (Ministerio de salud, 2015). Siendo el porcentaje en Chengue más alto al encontrado en las víctimas y familiares del 11 de marzo en Madrid $(15,4 \%)$ (Tobal, et al. 2005) e incluso que el presentado por personas que viven en cercanías al World Trade Center de NY, USA después del atentado a las torres gemelas (20\%) (Galea, et al., citado por Tobal, et al., 2005). Es interesante observar como en la población de Bojayá, específicamente en las personas que luego del desplazamiento no retornaron a su lugar de origen, no se encontró personas con presencia de TEPT (Londoño, et al, 2005), en contraste con la gente de Chengue que se quedó radicada en Ovejas que si presenta un alto porcentaje (26,2\%); igualmente difiere lo encontrado con otro tipo de desplazados evaluados en la ciudad de Sincelejo, los cuales no presenciaron actos violentos directamente, pero si fueron desplazados por la violencia, en los cuales se encontró el 5,5\% (Echenique, et al., 2008). Sin embargo, es de anotar que no supera lo hallado por Botelho y Conde (2011) en pacientes con TEPT victimas de desplazamiento, ya que encontraron el $57,9 \%$ con riesgo de tener el trastorno.

En la ansiedad generalizada (TAG) se encuentra que la población presenta 7 puntos porcentuales aproximadamente más alto que lo presentado en Colombia en la población adulta $(19,3 \%)$ y en la costa caribe lo supera en 12 puntos porcentuales (14,7\%) (Posada, et al., 2004). Sin embargo, Este porcentaje es más bajo que el encontrado en Bojayá (43\%) (Londoño, et al, 2005). En la mayoría de las entrevistas se encuentra que los problemas de ansiedad se asocian a las dificultades socioeconómicas y a las limitaciones 
para responder a las necesidades básicas de la familia y los hijos. Esta información es similar a la hallada por investigaciones anteriores, en las que se relacionan acontecimientos estresantes con psicopatología, especialmente con la aparición del TEPT (Buendía \& Mira, 1993; Falla, Chávez \& Molano, 2005; Juárez y Guerra, 2011; Martín \& Ochotorena, 2004; Médicos sin fronteras, 2006; Palacios \& Heinze, 2002; Santacruz \& Ardila, 2002).

Ahora bien, el trastorno de depresión mayor es el de la taza porcentual más alta del estudio (39,3\%); en Colombia se encontró en el 2004, en el estudio de Gómez, et al., que la población colombiana adulta presenta 10,2\% y en el estudio de 2015 (Ministerio de salud) hablan de 1,9\%. Se encontró un porcentaje similar en el estudio realizado en Bojayá (Londoño, et al., 2005), el cual fue de $37 \%$; esto podría comprenderse a partir de que ambas poblaciones vivieron un acontecimiento que generó la muerte de un gran número de miembros de la comunidad y a partir de allí se generó el desplazamiento.

Según Alejo (2005), al analizar el impacto psicológico del desplazamiento se debe considerar el hecho mismo que origina la decisión de partir, el impacto de los acontecimientos previos, el impacto del desplazamiento como tal y el impacto de las vivencias posteriores a éste. Esto es de vital importancia tenerlo en cuenta en el contexto colombiano, puesto que muchas personas antes de migrar vivieron acontecimientos sumamente estresantes tales como, masacres, torturas y hechos violentos en general que pudieron terminar desencadenando los primeros síntomas de los trastornos emocionales.

Llegar a otra población, muchas veces con costumbres diferentes y con la incapacidad para garantizar el bienestar a las familias, puede ser en alto grado estresante para los individuos que lo viven (Alejo, 2005). De hecho, una forma de la poca garantía de bienestar se refleja en el acceso a los servicios de salud. Moreno, et al. (2009) detectaron que los desplazados tienen peor accesibilidad a los servicios de salud y una mayor negación de este servicio por la ausencia de contratos. El 75\% de la población desplazada que participó en su estudio manifestó que no se les dio solución a sus necesidades de salud. El estudio de Moreno et al (2009) dio cuenta de una realidad que aún hoy sigue estando vigente, los derechos de los desplazados son desconocidos por un gran número de personas, especialmente para los mismos desplazados.

Lozano y Gómez (2004) afirman que la incertidumbre puede relacionarse con altos grados de estrés en las personas desplazadas. Estos indi- 
viduos no pueden proyectar un futuro próximo y esto puede influir en la generación de la incertidumbre que a su vez puede dar como consecuencia la experiencia de preocupación y de ansiedad.

A partir de los hallazgos de este estudio, podemos concluir que la situación de desplazamiento parece conllevar más acontecimientos vitales estresantes puesto que implica situaciones de estrés antes y después del desplazamiento, sin una debida red de apoyo y con más condiciones de precariedad en su nueva vida. También, se suma la experiencia vivida en la masacre, la cual generó altos niveles de estrés.

En cualquier caso, sería interesante llevar a cabo más estudios de este tipo, sobre todo, teniendo en cuenta la variable de red social, que no hemos estudiado en esta investigación y la variable de percepción de futuro, pues investigaciones anteriores (Lozano y Gómez, 2004) aseguran que esta variable produce muchos niveles de estrés en individuos desplazados.

\section{REFERENCIAS BIBLIOGRÁFICAS}

Alejo, E. G. (2005). Aplicación del modelo de estrés postraumático en el estudio del impacto de la violencia sobre la salud mental en población desplazada. MebUNAB, 8(1), $23-28$.

American Psychiatric Association (2000). DSM-IV-TR. Diagnostic and statistical manual of mental disorders (4thEdition Reviewed). Washington, DC: Author.

American Psychiatric Association (2013). DSM-5. Diagnostic and statistical manual of mental disorders (5thEdition). Washington, DC: Author.

Botelho, O. S., Conde, C. A. (2011). Memoria emocional y trastorno por estrés postraumático en el contexto del desplazamiento. Revista Colombiana de Psiquiatría, 40(3), 457-469.

Buendía, J., \& Mira, J. M. (1993). Estrés y Psicopatología. Madrid, España: Pirámide. Comisión Interamericana de Derechos Humanos. (2007). Informe n 45/07. Petición 1268-05 admisibilidad masacre de Chengue Colombia, 23 de julio.

Deng, F. (1998). Principios rectores de los desplazados internos (DOC.E/CN.4/ 1998/53/ADD.2). Ginebra, Suiza: Naciones Unidas.

Echenique, C., Medina, L. M., Medina, A., Ramírez, A. (2008). Prevalencia del trastorno por estrés postraumático en población desplazada por violencia, en proceso de restablecimiento. Psicología desde el Caribe, 21, 122-135.

Falla, U., Chávez, Y. A., \& Molano, G. (2005). Desplazamiento forzado en Colombia. Análisis documental e informe de investigación en la Unidad de Atención Integral al Desplazado (UAID)-Bogotá. Tabula Rasa, 1, 221-236.

Gómez-Restrepo, C., Bohórquez, A., Pinto, D., Laverde, J.F., Rondón, M., Díaz, N. (2004). Prevalencia de depresión y factores asociados con ella en la población colombiana. Rev. Panamericana de Salud Pública, 16, 378-386.

Gómez, P. (2001). Colombia y su salud mental. Bogotá, Colombia: Asociación colombiana de psiquiatría. 
Estado de salud mental de personas víctimas del conflicto armado.

Caso Chengue, Sucre-Colombia

Juárez, F., \& Guerra, A. (2011). Características Socioeconómicas y Salud en Personas Pobres y Desplazadas. Psicologia: Teoria e PesquisaOut-Dez, 27(4), 511-519.

Londoño, N., Muñiz, O. \& Correa, J.E. (2005). Salud mental en víctimas de la violencia armada en Bojayá (Chocó, Colombia). Revista colombiana de psiquiatría, 34, (4), 493-505.

Lozano, M. C., \& Gómez, M. L. (2004). Aspectos psicológicos, sociales y jurídicos del desplazamiento forzoso en Colombia. Acta colombiana de psicología, 12, $103-119$.

Martín, J. L., \& Ochotorena, J. P. (2004). Trastorno por estrés postraumático en víctimas de situaciones traumáticas. Psicothema, 16(1), 45-49.

Médicos Sin Fronteras (2006). Vivir con Miedo. El ciclo de la violencia en Colombia. Amsterdam, The Netherlands: Oficina de Asuntos Humanitarios MSF.

Ministerio de Salud. (2015). Encuesta nacional de salud mental 2015. Recuperado de http://www.visiondiweb.com/insight/lecturas/Encuesta_Nacional_de_Salud_Mental_Tomo_I.pdf

Ministerio de Protección Social. Fundación FES Social. (2005). Estudio Nacional de Salud Mental-Colombia 2003, ISBN 95833-73-70-2, recuperado de http:// onsm.ces.edu.co/uploads/files/1243030_EstudioNacionalSM2003.pdf.

Moreno, G. A., Monsalve, J. C., Tabima, D. G., \& Escobar, M. J. (2009). Apreciaciones de la población en condición de desplazamiento forzado sobre los servicios de salud en algunos municipios de Colombia. Rev Fac Nac Salud Pública, 27 (2), 131-141.

Palacio J, Mandariaga C, Sabatier C. (2001). Estrés post-traumático en jóvenes desplazados por la violencia política en Colombia. Disponible en URL:http:// interquisis.com

Palacios, L., \& Heinze, G. (2002). Trastorno por estrés postraumático, una revisión del tema (Primera parte). Salud Mental, 25(3), 19-26.

Posada, J., Aguilar, S., Magaña, C. \& Gómez, L.C. (2004). Prevalencia de trastornos mentales y uso de servicios: resultados preliminares del Estudio nacional de salud mental. Colombia, 2003. Revista colombiana de psiquiatría, 33 (3), 241-262.

Registro único de víctimas. (2015). Red nacional de información. Información al servicio de las víctimas. Consultado: el 01 de julio de 2015. http://rni.unidadvictimas.gov.co/?q=node/107

Sacipa, S. (2003). Lectura de los significados en historias del desplazamiento y una organización comunitaria por la paz. Universitas Psychologica, 2(1), 49-65.

Santacruz, H., \& Ardila, S. (2002). Fundamentos de Psiquiatría Clínica: Niños, Adolescentes y Adultos. (1a Ed.). Bogotá, Colombia: CEJA.

Silove D, Steel Z, McGorry P, Mohan P. (1998). Trauma exposure, postmigration stressors, and symptoms of anxiety, depression and post-traumatic stress in Tamil asylum-seekers: comparison with refugees and immigrants. Acta Psychiatr Scand, 97, 175-81.

Sheehan, D.V., Lecrubier, Y., Harnett, K., Janavs, J., Weiller, E., Bonora, L.I., et al. (1997). Reliability and Validity of the MINI International europsychiatric Interview (M.I.N.I.): According to the SCID-P. European Psychiatry. 12, 232-241. 
Andrés Fernando Ramírez Giraldo - Olga Leonor Hernández Bustamante -

Kelly Romero Acosta - Eduardo Porras Mendoza

Sheehan, D.V., Lecrubier, Y., Harnett, K., Amorim, P., Janavs, J., Weiller, E., et al. (1998). The Mini International Neuropsychiatric Interview (M.I.N.I.): The Development and Validation of a Structured Diagnostic Psychiatric Interview. J. Clin Psychiatry, 59 (suppl 20), 22-33.

Tobal, J., Cano, A., Iruzrrizaga, I., Gonzalez, H., Muñoz, M. \& Casado, I. (2005). Evolución de las consecuencias psicológicas provocadas por los atentados del 11-M. Recuperado 25 de julio de 2008. http://www.ucm.es/info/seas/tep/ resultados_investigac_atentados_11-M.html.

Torres, Y. et al. (2012). Primer Estudio Poblacional de Salud Mental Medellín, 20112012. Medellín, Colombia. L. Vieco e Hijas Ltda. 